\title{
Perfil epidemiológico e clínico de pacientes com lesão por pressão após trauma raquimedular
}

\author{
Epidemiological and clinical profile of patients with pressure injury \\ after spinal cord trauma
}

\section{Perfil epidemiológico y clínico de pacientes con lesión por presión después de una lesión medular}

\begin{abstract}
Fabio Conceição dos Santos ${ }^{1, *}$, Marcelo Williams Oliveira de Souza ${ }^{2}$, Odenilce Vieira Pereira ${ }^{3}$, Ingrid Magali de Souza Pimentel ${ }^{2}$, Aline Maria Pereira Cruz Ramos ${ }^{4}$
\end{abstract}

ORCID IDS

Santos FC (D) https://orcid.org/0000-0002-1191-7063

Souza MWO (D) https://orcid.org/0000-0002-1907-7687

Pereira OV iD https://orcid.org/0000-0002-4698-6413

Pimentel IMS (D) https://orcid.org/0000-0003-1820-5496

Ramos AMPC (D) https://orcid.org/0000-0001-8812-2923
COMO CITAR

Santos FC; Souza MWO; Pereira OV; Pimentel IMS; Ramos AMPC. Perfil epidemiológico e clínico de pacientes com lesão por pressão após trauma raquimedular. ESTIMA, Braz. J. Enterostomal Ther., 17, 2019: e2719. https://doi.org/10.30886/estima.v17.773_PT

\section{RESUMO}

Introdução: A prevalência de lesão por pressão (LP) varia com o ambiente clínico e as características do paciente, assim, pacientes com traumatismo raquimedular (TRM) configuram-se entre aqueles com maior predisposição diante do tempo de internação, apresentando movimentos e sensibilidade prejudicados. Objetivo: Conhecer e analisar a prevalência de LP em pacientes vítimas de TRM internados em um hospital de referência. Método: Estudo transversal, retrospectivo. A amostra compõe-se de prontuários de pacientes com TRM que apresentaram LP durante a internação em um hospital de referência no estado do Pará, Brasil, durante o período de 2013 a 2016. Resultados: Analisaram-se 565 prontuários de pacientes com trauma raquimedular, com a prevalência de lesão por pressão em de 8\% dos pacientes com TRM. A média de idade foi de 37 anos, com 80\% composta por homens, com tempo médio de internação de 66,68 dias. A maioria apresentou como etiologia da lesão medular os acidentes por arma de fogo e queda, com 32 e 30\% respectivamente. A lesão medular foi predominante na região torácica (50\%), onde 64\% tiveram a paraplegia como sequela e 86\% apresentaram LP em região sacral. Conclusão: Foi possível identificar a prevalência de lesão por pressão em pacientes com traumatismo raquimedular e conhecer seu comportamento. Desse modo, a disponibilização dos dados ajudou a demostrar a ocorrência de LP como complicação em pacientes com TRM durante a internação, podendo servir como base para a alocação de recursos e medidas preventivas, como também estudos posteriores sobre a temática.

DESCRITORES: Coluna vertebral; Lesão por pressão; Epidemiologia; Estomaterapia.

\begin{abstract}
Introduction: The prevalence of pressure injury (PI) varies with the clinical environment and the characteristics of the patient, thus, patients with spinal cord trauma (SCT) are configured among those with greater predisposition to the length of hospitalization, presenting impaired movements and sensitivity. Objective: To know and analyze the prevalence of pressure injury in patients suffering from spinal cord trauma hospitalized in a reference hospital. Method: Cross-sectional, retrospective study. The sample consists of medical records of patients with spinal cord trauma who presented pressure injury during hospitalization in a reference
\end{abstract}

1.Centro Universitário do Estado do Pará - Programa de Residência Multiprofissional em Neurologia - Belém/PA - Brasil.

2.Universidade do Estado do Pará - Escola de Enfermagem Magalhães Barata - Departamento de Enfermagem Hospitalar - Belém/PA - Brasil.

3.Unidade Hospitalar João de Barros Barreto - Departamento de Enfermagem - Belém/PA - Brasil.

4. Universidade Federal do Pará - Faculdade de Enfermagem - Belém/PA - Brasil.

*Autor correspondente: fabiosantos-166@hotmail.com

Recebido: Jun. 23, 2019 | Aceito: Nov. 13, 2019 
hospital in the state of Pará, Brazil, during the period from 2013 to 2016. Results: We analyzed 565 medical records of patients with spinal cord, with the prevalence of PI in of $8 \%$ of patients with SCT. The mean age was 37 years, with $80 \%$ composed of men, with an average length of hospitalization of 66.68 days. The majority presented as etiology of spinal cord injury accidents by firearm and fall, with 32 and 30\% respectively. Spinal cord injury was predominant in the thoracic region (50\%), where 64\% had paraplegia as sequelae and $86 \%$ presented pressure injury in the sacral region. Conclusion: It was possible to identify the prevalence of PI in patients with SCT and to know its behavior. Thus, the availability of data helped to demonstrate the occurrence of PI as a complication in patients with SCT during hospitalization, and can serve as a basis for the allocation of preventive resources and measures, as well as further studies on the theme.

DESCRIPTORS: Spine; Pressure injury; Epidemiology; Enterostomal therapy.

\section{RESUMEN}

Introducción: La prevalencia de la lesión a presión varía según el entorno clínico y las características del paciente, por lo tanto, los pacientes con Trauma de la Médula Espinal (TME) se configuran entre aquellos con mayor predisposición a la duración de la hospitalización, presentando movimientos y sensibilidad deteriorados. Objetivo: Conocer y analizar la prevalencia de lesión por presión en pacientes que sufren de traumatismo de la médula espinal hospitalizados en un hospital de referencia. Método: Estudio transversal retrospectivo. La muestra consiste en registros médicos de pacientes con traumatismo de la médula espinal que presentaron lesión por presión durante la hospitalización en un hospital de referencia en el estado de Pará, Brasil, durante el período de 2013 a 2016. Resultados: Analizamos 565 registros médicos de pacientes con médula espinal, con la prevalencia de Lesión por Presión en pacientes con traumatismo de la médula espinal del 8\%. La edad media fue de 37 años, con un 80\% compuesto por hombres, con una duración media de hospitalización de 66,68 días. La mayoría se presentó como etiología de accidentes de lesión de la médula espinal por arma de fuego y caída, con 32 y 30\% respectivamente. La lesión de la médula espinal fue predominante en la región torácica (50\%), donde el 64\% tenía paraplejia como secuencias y el 86\% presentó lesión por presión en la región sacral. Conclusión: Fue posible identificar la prevalencia de lesión por presión en pacientes con traumatismo de la médula espinal y conocer su comportamiento. Por lo tanto, la disponibilidad de datos ayudó a demostrar la aparición de LP como una complicación en pacientes con TME durante la hospitalización, y puede servir de base para la asignación de recursos y medidas preventivas, así como más estudios sobre el tema.

DESCRIPTORES: Columna vertebral; Lesión por presión; Epidemiología; Estomaterapia.

\section{INTRODUÇÃO}

O aumento na expectativa de vida é decorrente também de avanços na assistência à saúde, tornando possível a sobrevida de pacientes com doenças graves, crônicas e debilitantes, repercutindo-se em maior tempo de internação e surgimento de complicações, como a lesão por pressão $(\mathrm{LP})^{1}$. A ocorrência dessa lesão varia de acordo com o ambiente clínico e as características do paciente, com maior frequência nos pacientes agudamente hospitalizados ou nas longas internações².

Assim sendo, os pacientes com traumatismo raquimedular (TRM) se configuram entre aqueles com maior predisposição a LP diante do prolongado período de internação, pois permanecem acamados com sensibilidade e movimentos prejudicados. Além disso, o TRM pode cursar com o aparecimento de outras complicações e comprometimentos indiretos, tais como disreflexia autonômica, hipotensão postural, ossificação heterotópica, contraturas, trombose venosa profunda, síndromes dolorosas (dor traumática, dor na raiz nervosa, entre outras), osteoporose e cálculos renais ${ }^{3}$.

No Brasil, estima-se que ocorram cerca de 40 novos casos de TRM por milhão de habitantes, com total de 6 a 8 mil casos por ano, dentre os quais, $80 \%$ das vítimas são homens, representando custo elevado ao sistema de saúde. Ressalta-se que os dados não possuem exatidão devido à não obrigatoriedade de notificação ${ }^{4}$.

Em pacientes hospitalizados o desenvolvimento de LP constitui um grande problema de saúde, pois pode acarretar desconforto físico, aumento do risco de complicações adicionais, prolongamento da hospitalização e elevação de custos relacionados ao tratamento 5 .

Segundo a National Pressure Ulcer Advisory Panel (NPUAP), lesão por pressão é um dano localizado na pele e/ou tecido mole subjacente geralmente sobre uma proeminência óssea ou está relacionada ao uso de dispositivo médico. A lesão decorre do resultado da pressão intensa e/ou prolongada em combinação com o cisalhamento, podendo ainda ser afetada pelo microclima, nutrição, perfusão, comorbidades e pela sua condição, além de caracterizar um indicador negativo da qualidade do cuidado ${ }^{2}$.

Trata-se de uma lesão multifatorial cuja assistência é um desafio para a equipe de enfermagem, considerando a complexidade dos cuidados. Entretanto essa categoria tem implementado medidas preventivas sistematizadas em protocolos baseados em diretrizes internacionais ${ }^{5}$. Consoante a isso, os gestores dos serviços de saúde têm se preocupado com a temática relacionada 
à ocorrência desse evento, em função dos aspectos éticos, sociais, legais e financeiros, o que determina implementar medidas de prevenção e os indicadores de qualidade relacionados a esse agravo, haja vista que a redução do risco de LP é uma das metas dentro do Programa Nacional de Segurança do Paciente 6 .

Diante disso, descrever e analisar a ocorrência de LP em pacientes com TRM é fundamental a fim de contribuir para estimativas epidemiológicas, para que haja um delineamento de futuras intervenções capazes de diminuir seu aparecimento e complicações, além da diminuição dos custos com o tratamento da LP quando instalada. Assim, essa pesquisa torna-se pioneira em nossa região e relevante na medida em que possa subsidiar as práticas de cuidado em saúde como maneira de prevenir e/ ou minimizar o surgimento de LP em pacientes com TRM.

Portanto, considerando a justificativa para os estudos científicos da problemática apresentada, emergiu a inquietação acerca desta pesquisa, a qual se norteou pela seguinte pergunta: qual a ocorrência de lesão por pressão em pacientes portadores de lesão medular em pacientes vítimas de trauma raquimedular em um hospital de referência?

\section{OBJETIVO}

Conhecer e analisar a prevalência de lesão por pressão em pacientes vítimas de trauma raquimedular internados em um hospital referência no estado do Pará.

\section{MÉTODO}

Trata-se de um estudo transversal, retrospectivo. $\mathrm{O}$ estudo foi realizado em um hospital de urgência e emergência localizado na região metropolitana de Belém, estado do Pará, Brasil. Essa instituição é referência no atendimento às vítimas de trauma e queimaduras, atendendo a média e alta complexidade.

A coleta de dados procedeu-se no serviço de arquivo médico e estatístico do referido hospital, após autorização. Foram utilizados dados secundários (prontuários finalizados) dos pacientes que sofreram acidentes que resultaram em trauma raquimedular durante o período de quatro anos, entre janeiro de 2013 a dezembro de 2016. Foram elegíveis os prontuários de pacientes com idades de 18 a 80 anos, com diagnóstico de TRM e que desenvolveram LP durante a internação. Foram excluídos os prontuários de pacientes menores de 18 e acima de 80 anos, com LP na admissão, e aqueles fora do período estabelecido para a pesquisa. Ao total fizeram parte do estudo 565 prontuários, sendo 112 em 2013,128 em 2014, 179 em 2015 e 146 em 2016.

Para a coleta dos dados, utilizou-se um formulário confeccionado pelos autores com perguntas fechadas e as variáveis: 1) sociodemográficas: período de internação, idade, sexo, naturalidade e município de origem, alta ou óbito;2) dados clínicos do TRM: etiologia, nível da lesão medular, sequela; 3) dados da LP: tempo para primeira lesão, número de lesões apresentadas, regiões acometidas, presença ou não de infecção e se houve, qual foi; 4) dados sobre os cuidados de enfermagem: cuidados preventivos, diagnósticos e assistência de enfermagem ao paciente com LP.

Os dados foram tabulados no software Microsoft Office Excel 2016, submetido à estatística descritiva com cálculo das variáveis (frequência absoluta, relativa, desvio padrão e prevalência de LP nos pacientes por ano e no período em estudo) propostas em tabelas e gráficos, com o programa software BioEstat 5.3. Para identificar a prevalência da LP foi realizado o cálculo matemático: número de paciente que apresentaram $\mathrm{LP}$ em cada ano dividido pelo total de pacientes com TRM por ano, sendo cada resultado multiplicado por 100.Dessa mesma maneira realizou-se o cálculo da prevalência durante o período de 2013 a 2016: número de pacientes que apresentaram LP de 2013 a 2016 dividido pelo total de pacientes com TRM de 2013 a 2016, multiplicando o valor final por 100. Os dados foram interpretados e feita discussão com os achados na literatura, dando aporte ao conteúdo.

O estudo seguiu as diretrizes estabelecidas pela resolução n 466 de dezembro de 2012 do Conselho Nacional de Saúde. O projeto teve aprovação do Comitê de Ética e Pesquisa em Seres Humanos - CEP do Centro Universitário do Estado do Pará - CESUPA, sob número de parecer 2.683.731 e CAAE 90029218.0.0000.5169.

\section{RESULTADOS}

As características sociodemográficas e clínicas dos pacientes serão aqui apresentadas em tabelas. Do total de 565 prontuários pesquisados, 50 (8,84\%) pacientes com TRM desenvolveram LP durante a hospitalização entre o período de janeiro de 2013 a dezembro de 2016. A seguir os dados anual dessa lesão, assim como sua prevalência anual e durante o período (Tabela 1 ).

$\mathrm{Na}$ Tabela 2, verifica-se que a média de idade dos pacientes foi 37,08 $( \pm 15,33)$ anos, apresentando idade máxima de 80 anos e mínima de 18 anos. $O$ gênero masculino predominou entre os pacientes pesquisados em relação ao feminino com 40 (80\%) e 
Tabela 1. Distribuição dos casos de trauma raquimedular (TRM) e lesão por pressão (LP) pós-TRM, de acordo com o ano, em pacientes atendidos em um hospital de referência, de 2013 a 2016. Belém-PA, 2019.

\begin{tabular}{cccc}
\hline $\begin{array}{c}\text { Ano de } \\
\text { ocorrência }\end{array}$ & $\begin{array}{c}\text { Casos de } \\
\text { TRM }\end{array}$ & $N^{\circ}$ de LP & $\begin{array}{c}\text { Prevalência } \\
\text { de LP }\end{array}$ \\
\hline 2013 & 112 & 6 & 5 \\
\hline 2014 & 128 & 13 & 10 \\
\hline 2015 & 179 & 14 & 7 \\
\hline 2016 & 146 & 17 & 11 \\
\hline Total & 565 & 50 & 8 \\
\hline
\end{tabular}

Fonte: Dados da pesquisa, 2019.

10 (20\%) respectivamente. Observou-se que 21 pacientes (42\%) eram da região metropolitana de Belém e 29 (58\%) do interior do estado. O tempo médio de internação foi de 66,68 dias, com máximo de 161 e mínimo de 5 dias. O desfecho clínico da maioria dos pacientes foi: 44 (88\%) alta melhorada e $6(12 \%)$ de óbitos por causas diversas, com destaque para sepse, pneumonia associada à ventilação mecânica, tromboembolismo venoso e outras complicações.

Sobre os aspectos clínicos dos pacientes com TRM (Tabela $3)$, evidenciou-se que 16 pacientes (32\%) apresentaram como causa do trauma os acidentes por arma de fogo e 15 (30\%) por queda. Em relação à região do corpo que mais foi afetada pelo trauma, predominou a região torácica com 24 dos casos (50\%), seguidos das regiões cervical com 13 (26\%) e lombar com 20 (10\%). Sabe-se que o comprometimento do nível medular na coluna determina as sequelas irreversíveis. Nesta pesquisa, 32 (64\%) evoluíram para paraplegia e 18 (36\%) para tetraplegia.

Quanto às características das LPs (Tabela 4) relacionadas ao aparecimento, fatores de risco, número de lesões, região do corpo afetada e presença de infecção, foram evidenciados os seguintes dados: tempo médio de aparecimento foi de 19,32 dias; entre os fatores de risco destacou-se o tabagismo associado ao etilismo em 13 casos (26\%) da amostra, sendo que 21 lesões (42\%) estiveram associadas a outras comorbidades (perda da função motora e sensibilidade, desnutrição, hipertensão, incontinência fecal e urinária, entre outros). Vale ressaltar que 35 pacientes (70\%) desenvolveram apenas uma LP, com média em torno de 1,68 ( $\pm 1,16)$; em contrapartida, 7 pacientes $(14 \%)$ apresentaram três LPs. Quanto à área corporal mais afetada, houve predomínio da região sacral com 43 ocorrências (86\%), seguida do calcâneo e região occipital com 3 (6\%) ocorrências em cada local. Sobre a infecção das lesões, somente 15 pacientes (30\%) do total de prontuários analisados, apresentaram LP infectada, todas na região sacral.
Tabela 2. Perfil sociodemográfico de pacientes com TRM que apresentaram LP em um hospital de referência, de 2013 a 2016. Belém-PA, 2019.

\begin{tabular}{|c|c|c|}
\hline Características & $n=50$ & Freq. (\%) \\
\hline \multicolumn{3}{|l|}{ Gênero } \\
\hline Masculino & 40 & 80 \\
\hline Feminino & 10 & 20 \\
\hline \multicolumn{3}{|l|}{ Naturalidade } \\
\hline Pará & 50 & 100 \\
\hline Outro estado & 0 & 0 \\
\hline \multicolumn{3}{|l|}{ Municípios de origem } \\
\hline Região metropolitana & 21 & 42 \\
\hline Outros municípios & 29 & 58 \\
\hline $\begin{array}{l}\text { Tempo médio de internação (máximo } \\
161 \text { dias e mínimo } 5 \text { dias) }\end{array}$ & 66,68 & \\
\hline \multicolumn{3}{|l|}{ Desfecho clínico } \\
\hline Alta melhorada & 44 & 88 \\
\hline Óbito & 6 & 12 \\
\hline
\end{tabular}

Fonte: Dados da pesquisa, 2019.

Tabela 3. Aspectos clínicos relacionados ao TRM dos pacientes investigados que apresentaram LP em um hospital de referência, de 2013 a 2016. Belém-PA, 2019.

\begin{tabular}{|c|c|c|}
\hline Características & $\mathrm{n}=50$ & Freq. (\%) \\
\hline Acidente de carro & 3 & 6 \\
\hline Acidente de moto & 7 & 14 \\
\hline Acidente por arma de fogo & 16 & 32 \\
\hline Queda & 15 & 30 \\
\hline Mergulho em águas rasas & 2 & 4 \\
\hline Outros & 7 & 14 \\
\hline \multicolumn{3}{|l|}{ Nível de lesão } \\
\hline Cervical & 13 & 26 \\
\hline Torácica & 25 & 50 \\
\hline Lombar & 10 & 20 \\
\hline Cérvico-torácica & 1 & 2 \\
\hline Torácica-lombar & 1 & 2 \\
\hline \multicolumn{3}{|l|}{ Sequela apresentada } \\
\hline Paraplegia & 32 & 64 \\
\hline Tetraplegia & 18 & 36 \\
\hline
\end{tabular}

Fonte: Dados da pesquisa, 2019

$\mathrm{Na}$ Tabela 5 apresentam-se os cuidados preventivos realizados pela equipe de enfermagem aos pacientes com TRM. Dos 50 (100\%) prontuários analisados, identificou-se como cuidados prioritários: instalação de colchão piramidal (100\%), mudança de decúbito (96\%), uso de coxins e manutenção do paciente isento de umidade (80\%), como prevenção às LPs. 
Tabela 4. Características relacionadas a LP dos pacientes com TRM em um hospital de referência, de 2013 a 2016. Belém-PA, 2019.

\section{Características}

Freq.

Média do tempo para $1^{\text {a }}$ LP em dias e desvio padrão

Fatores de risco

Tabagismo

$19,32 \pm 17,23$

Diabetes

Doença cardiovascular

$6 \quad 12$

Alcoolismo

Tabagismo + etilismo

Diabetes + etilismo

Diabetes + tabagismo

36

120

24

$13 \quad 26$

24

Outros (perda da função motora e sensibilidade, desnutrição, hipertensão, incontinência fecal

e urinária, entre outros)

21

42

Números de lesões

Média de LP por paciente e desvio padrão

$1,68 \pm 1,16$

\begin{tabular}{|c|c|c|}
\hline Uma & 35 & 70 \\
\hline Duas & 3 & 6 \\
\hline Três & 7 & 14 \\
\hline Quatro & 3 & 6 \\
\hline Cinco & 2 & 4 \\
\hline \multicolumn{3}{|c|}{ Região em que ocorreu a primeira LP } \\
\hline Occipital & 3 & 6 \\
\hline Sacral & 43 & 86 \\
\hline Calcanhar & 3 & 6 \\
\hline Outra & 1 & 2 \\
\hline \multicolumn{3}{|l|}{ Presença de lesão infectada } \\
\hline Sim (todas na região sacral) & 15 & 30 \\
\hline Não & 35 & 70 \\
\hline
\end{tabular}

Fonte: Dados da pesquisa, 2019

Tabela 5. Cuidados preventivos à LP e DE em pacientes com TRM prestados pela equipe de enfermagem em um hospital de referência, de 2013 a 2016. Belém-PA, 2019.

\begin{tabular}{|c|c|c|c|c|c|}
\hline Cuidados preventivos & Freq. & $\%$ & Diagnósticos de enfermagem & Freq. & $\%$ \\
\hline Uso de colchão piramidal & 50 & 100 & Integridade da pele prejudicada & 50 & 100 \\
\hline Mudança de decúbito & 48 & 96 & Risco de infecção & 50 & 100 \\
\hline Uso de coxins & 40 & 80 & Mobilidade física prejudicada & 50 & 100 \\
\hline Manutenção do paciente isento de umidade & 40 & 80 & Risco de queda & 50 & 100 \\
\hline Uso de creme barreira & 35 & 70 & Mobilidade no leito prejudicada & 45 & 90 \\
\hline Manter lençóis secos e esticados & 34 & 68 & Dor aguda & 44 & 88 \\
\hline Observar, anotar e comunicar alterações cutâneas & 33 & 66 & Déficit no autocuidado para banho/higiene & 42 & 84 \\
\hline Uso de filmes transparentes em região de proeminências & 25 & 50 & Perfusão tissular ineficaz & 38 & 76 \\
\hline \multirow[t]{3}{*}{ Higiene íntima após eliminações } & 15 & 30 & Conforto prejudicado & 38 & 76 \\
\hline & & & Ansiedade & 27 & 54 \\
\hline & & & $\begin{array}{l}\text { Nutrição desequilibrada, menor que as } \\
\text { necessidades corporais }\end{array}$ & 10 & 20 \\
\hline
\end{tabular}

Fonte: Dados da pesquisa, 2019. 
Os diagnósticos de enfermagem (DE) estabelecidos aos pacientes com LP pós-TRM também são descritos. Evidenciou-se que cerca de $100 \%$ da amostra cursaram com: integridade da pele prejudicada, risco de infecção, mobilidade física prejudicada e risco de queda.

\section{DISCUSSÃO}

Este estudo evidenciou a LP como uma das complicações desenvolvidas por pacientes com TRM, considerando que a lesão medular é uma das mais graves complicações que causa incapacidade ao ser humano, comprometendo funções vitais, dentre as quais a locomoção e perda da sensibilidade. Estudos semelhantes a este abordam prevalência de LP nesses pacientes e que o acometimento do paciente com lesão medular pela LP impacta fortemente na qualidade de vida dessas pessoa ${ }^{7,8}$.

O cometimento do TRM em homens ainda é maior que em mulheres, como em pesquisa semelhante a esta, em que se evidenciou que homens foram os mais acometidos pelo TRM. Esse perfil pode estar relacionado ao estilo de vida, pois homens exibem comportamento desafiador ao se exporem mais às ocasiões de risco?.

Com relação a outras variáveis sociodemográficas dos pacientes com TRM, evidenciou-se pacientes com idades em torno de 37 anos, provenientes da área metropolitana ou interior do estado, com tempo médio de internação acima de 60 dias diante das complicações, dentre elas a $\mathrm{LP}^{10}$. Ainda, sobre à procedência dos pacientes, vale ressaltar que Pereira, Gomes e Rodrigues relataram em estudo semelhante que muitos dos pacientes são transferidos para as grandes cidades, pela falta de estrutura e leitos para atendimento ao politraumatizado ${ }^{10}$.

É importante apontar que a taxa de óbito neste estudo não foi elevada, assim, os pacientes que receberam alta melhorada e retornaram aos seus lares com algum tipo de necessidade especial permanente, por conseguinte, irão por vezes necessitar de cuidados especializados, não exequíveis diante da condição social ou pela ausência dos serviços de referência e reabilitação ${ }^{10,11}$.

Os seguimentos medulares mais acometidos são as regiões cervical e torácica, causados por acidentes por arma de fogo, seguidos dos acidentes oriundos de quedas e mergulho em águas rasas ${ }^{10,12,13}$, estando de acordo com os achados nesta pesquisa. $\mathrm{O}$ trauma medular total do seguimento cervical e a parte inicial da coluna torácica pode trazer como consequência a tetraplegia e, em outras situações, a dissecção parcial de outras partes da coluna trará como sequela a paraplegia conforme estudo verificado ${ }^{14} \mathrm{e}$ averiguado nesta investigação.

O desenvolvimento de uma LP é multifatorial e os pacientes com TRM, críticos em sua maioria, evoluem com a instabilidade hemodinâmica e mobilidade prejudicada que o restringe ao leito, o que favorece o desenvolvimento da LP. Alguns autores evidenciam que paciente críticos apresentam algum risco para o desenvolvimento de LP após 2 ou 3 dias ${ }^{15}$.

A segurança do paciente tem relação direta com a redução de riscos e danos evitáveis ao mínimo possível na assistência em saúde, logo, o aumento de tempo na internação resulta em lesões na pele, consideradas um problema de saúde grave e preocupante. Ainda, a ocorrência de LP no âmbito hospitalar é considerada como evento adverso e pode retratar a qualidade da assistência prestada pela instituição de saúde ${ }^{16,17}$.

No que se refere ao número de LP apresentada por paciente, a literatura aponta que a média é de 1,85 lesão por paciente, predominantemente na região sacral e trocânter, fato evidenciado em nossa pesquisa, onde a maioria dos pacientes apresentou LP na região sacral. Isso se justifica pela funcionalidade das estruturas ósseas de apoio do paciente, seja sentado, ou em decúbito dorsal horizontal ou lateral, bem como a interface desses ossos longos com inúmeros músculos ${ }^{18}$.

A prevenção de LP tem sido considerada um indicador de qualidade não só da unidade/serviço de saúde, como também dos cuidados de enfermagem. Diante disso, a equipe de enfermagem tem implementado intervenções preventivas a LP, embora se reconheça a sua multicausalidade. A literatura destaca que a implementação sistemática das melhores práticas, recomendadas nas diretrizes para a prática clínica tem sido um desafio para muitas instituições ${ }^{19}$.

As medidas de prevenção não se diferem muito das encontradas neste estudo, porém incitam a repensar sobre a prática assistencial de enfermagem, que deve ser pautada em evidências científicas que reflitam no cuidado ao paciente.

No Brasil, um estudo realizado para verificar a ocorrência da LP nos pacientes com lesão medular acentuou as intervenções para a prevenção. Essa mesma preocupação também é pontuada pelos enfermeiros ao considerarem o processo de enfermagem voltado para o olhar clínico sobre o paciente como essencial, no que tange à prevenção e aos cuidados voltados especificamente para pacientes com LP $\mathrm{P}^{20,21}$. 
Como limitações do estudo, os autores tiveram o tempo mínimo disponibilizado pela instituição de saúde para a coletados dos dados e a falta de informações coerentes referentes ao diagnóstico de TRM e tempo de aparecimento da LP nos pacientes.

\section{CONCLUSÃO}

A pesquisa proporcionou o conhecimento do perfil de pacientes com trauma raquimedular (TRM) que desenvolveram lesão por pressão (LP) durante o período de internação em um hospital de referência localizado na região metropolitana de Belém-Pará, evidenciando que a maioria das vítimas do TRM são pessoas do sexo masculino, que permaneceram em média 66 dias internados, tiveram como etiologia da lesão medular os acidentes por arma de fogo e queda, respectivamente. Contudo a grande maioria recebeu alta melhorada, porém apresentando a paraplegia como sequela permanente.

O estudo ainda evidenciou que o nível medular mais acometido pelo trauma foram as regiões torácica e cervical. O tempo médio para o aparecimento da primeira LP foi de 19 dias, a maioria dos pacientes apresentou apenas uma LP e a região em que mais apareceu foi a sacral.
Além disso, conheceu-se os cuidados preventivos elencados pela equipe de enfermagem aos pacientes com TRM, como forma de redução dos riscos de LP. Nesse sentido, ressalta-se a necessidade do engajamento da equipe transdisciplinar, o envolvimento das lideranças das instituições de saúde, o treinamento para o profissional de enfermagem e a equipe para cuidados da pele e tratamento da LP quando instalada, assim como educação permanente e feedback dos resultados alcançados.

Portanto a disponibilização dos dados ajudou a demostrar a ocorrência de LP como complicação em pacientes com TRM durante a internação, podendo servir como base para a alocação de recursos e medidas preventivas, como também estudos posteriores sobre a temática.

\section{CONTRIBUIÇÃO DOS AUTORES}

Contextualização, Santos FCS e Ramos AMPC; Metodologia, Santos FCS e Ramos AMPC; Investigação, Santos FCS; Redação - Primeira versão, Santos FCS; Souza MWO; Pereira AOV; Pimentel IMS e Ramos AMPC; Redação - Revisão \& Edição, Santos FCS; Souza MWO; Pereira AOV; Pimentel IMS e Ramos AMPC; Recursos, Santos FCS e Souza MWO; Supervisão, Ramos AMPC.

\section{REFERÊNCIAS}

1. Soares CF, Heidemann ITSB. Promoção da saúde e prevenção da lesão por pressão: expectativas do enfermeiro da atenção primária. Texto Contexto Enferm. 2018;27(2):19. https://doi.org/10.1590/0104-070720180001630016

2. National Pressure Ulcer Advisory Panel-NPUAP. About us [Internet]. Washington: NPUAP; 2016: http://www.npuap. org/about-us/

3. Bomfim EO, Cabral DB, Júnior LCL, Santos MF, Cavalcante GM. Úlceras por pressão em pacientes com lesão medular traumática: subsídios na identificação microbiológica. J Res: Fundam Care Online 2014;6(2):747-58. https://doi. org/10.9789/2175-5361.2014v6n2p747

4. Rouanet C, Reges D, Rocha E, Gagliardi V, Silva GS. Traumatic spinal cord injury: current concepts and treatment update. Arq Neuropsiquiatr. 2017;75(6):387-93. https://doi. org/10.1590/0004-282X20170048

5. Lima AFC, Castilho V. Body mobilization for prevention of pressure ulcers: direct labor costs. Rev Bras Enferm. 2015;68(5):647-52. https://doi.org/10.1590/0034$7167.2015680523 i$
6. Mittag BF, Krause TCC, Roehrs H, Meier MJ, Danski MTR. Cuidados com lesão de pele: Ações da enfermagem. ESTIMA, Braz J Enterostomal Ther. 2017;15(1):19-25. https:// doi.org/10.5327/Z1806-3144201700010004

7. Zakrasek EC, Creasey G, Crew JD. Pressure ulcers in people with spinal cord injury in developing nations. Spinal Cord. 2015;53:7-13. https://doi.org/10.1038/sc.2014.179

8. Ministério da Saúde. Diretrizes de atenção ao lesado medular. 2 ed. Brasília (DF): Ministério da Saúde; 2015.

9. Bernardi DM. Epidemiologic profile of surgery for spinomedullary injury at a referral hospital in a country town of Brazil. Coluna/Columna. 2014;13(2):136-8. https:// doi.org/10.1590/S1808-18512014130200273

10. Pereira ELR, Gomes AL, Rodrigues DB. Epidemiologia do traumatismo Raquimedular por projéteis de armas de fogo em um hospital de referência no estado do Pará. Arq Bras Neurocir. 2015;34(1):13-9. https://doi. org/10.1055/s-0035-1547384

11. Carvajal C, Pacheco C, Gómez-Rojo C, Calderón J, Cadavid C, Jaimes F. Características clínicas y demográficas de 
pacientes con trauma Raquimedular Experiencia de seis años. Acta Med Colomb. 2015;40(1):45-50.

12. Silva OT, Ghizoni E, Tedeschi H, Joaquim AF. Epidemiology of spinal trauma surgically treated at the UNICAMP Hospital das Clínicas. Coluna/Columna. 2018;17(1):55-8. https://doi. org/10.1590/s1808-185120181701179262

13. Oliveira TAB, Andrade SMS, Prado GO, Fernandes RB, Gusmão MS, Gomes EGF, et al. Epidemiology of spine fractures in motorcycle accident victims. Coluna/Columna. 2016;15(1):65-67. https://doi.org/10.1590/S1808185120161501147147

14. Araujo AO, Ferronato DS, Rocha ID, Marcon RM, Alexandre CF, Filho TEPB. Profile of spinal cord trauma victims treated at a reference unit in São Paulo. Coluna/Columna. 2018;17(1):3941. https://doi.org/10.1590/S1808-185120181701178599

15. Otto C, Schumacher B, Wiese LPDL, Ferro C, Rodrigues RA. Fatores de risco para o desenvolvimento de lesão por pressão em pacientes críticos. Enferm Foco. 2019;10(1):0711. https://doi.org/10.21675/2357-707X.2019.v10.n1.1323
16. Martins M. Qualidade do cuidado em saúde. In: Sousa P, Mendes W, (Org.). Segurança do paciente: Conhecendo os riscos nas organizações de saúde. Rio de Janeiro (RJ): EAD/ ENSP; 2014. 25-38 p.

17. Fonseca $A D$, Peterlini $F L$, Costa DA, coordenadores. Segurança do paciente. São Paulo (SP): Martinari; 2014.

18. Mota, D, Barbosa Ribeiro, M. Calidad de vida en portadores de lesión medular con úlceras por presión. Enferm Glob. 2016;15(42):13-21.

19. Vasconcelos JMB, Caliri MHL. Ações de enfermagem antes e após um protocolo de prevenção de lesões por pressão em terapia intensiva. Esc Anna Nery. 2017;21(1):1-9. https://doi. org/10.5935/1414-8145.20170001

20. Andrade LT, Chianca TCM. Validação de intervenções de enfermagem para pacientes com lesão medular e mobilidade física prejudicada. Rev Bras Enferm. 2013;66(5):688-93. https://doi.org/10.1590/S0034-71672013000500008

21. Vasconcelos AS, França ISX, Sousa FS, Costa MML. Diagnósticos de enfermagem identificados no sujeito com lesão medular. J Nurs UFPE on-line. 7(5):1326-32. 